1971

\title{
Two-, Three-, and Four-Particle Spatial Correlations Among Tertiary Cosmic Ray Muons
}

David D. Busath

R O. Stevenson

Follow this and additional works at: https://scholarsarchive.byu.edu/facpub

Part of the Cell and Developmental Biology Commons, and the Physiology Commons

\section{Original Publication Citation}

Busath, D.D. and R.O. Stenerson. 1971. 2-particle, 3-particle and 4-particle spatial correlations among tertiary cosmic ray muons. Nuclear Physics B. 35:141-159.

\section{BYU ScholarsArchive Citation}

Busath, David D. and Stevenson, R O., "Two-, Three-, and Four-Particle Spatial Correlations Among Tertiary Cosmic Ray Muons" (1971). Faculty Publications. 1359.

https://scholarsarchive.byu.edu/facpub/1359 


\title{
TWO-, THREE-, AND FOUR-PARTICLE SPATIAL CORRELATIONS AMONG TERTIARY COSMIC RAY MUONS *
}

\author{
D.D. BUSATH and R.O. STENERSON \\ University of Utah, Salt Lake City, Utah 84112
}

Received 16 June 1971

(Revised 31 August 1971)

\begin{abstract}
Showers of muons which result from interactions of primary cosmic rays high in the atmosphere appear deep underground as bundles of nearly parallel muons. For threshold energies of $1 \mathrm{TeV}$ and arrival zenith angles of $\mathbf{4 5}$ degrees, those events having a small mean number of muons are described by a power law distribution in shower size having an exponent of $\simeq-3.7$ and a radial density distribution that decreases with distance $R$ from the shower axis a little less rapidly than $\exp (-R / 5.4 \mathrm{~m})$. Comparison of new data on four muon spatial correlations in terms of the same phenomenology with data on two and three muon spatial correlations from previous experiments suggests that the shower size distribution steepens and the shower radial density distribution broadens with increasing shower size (i.e., with increasing mean energy of the primary cosmic ray at a given threshold energy of the muons observed). These results might be explained by a basically geometrical effect due either to a total inelastic cross section which rises with energy or by an enhanced contribution from the interactions of secondaries. If the effect does not have a geometrical origin, then it may be necessary to reformulate the input to shower development calculations which have assumed that interaction products are described by factored, uncorrelated single particle statistical distributions. The relationships between the inclusive distributions predicted by theory and those of the empirical phenomenology are explored in.some detail.
\end{abstract}

\section{INTRODUCTION}

Muon cores of air showers produced by interactions of primary cosmic rays high in the atmosphere appear deep underground as nearly parallel bundles of penetrating muons. From the fact that observed showers having individual muons with threshold energies above $1 \mathrm{TeV}$ have characteristic radii of the order of $5 \mathrm{~m}$ and that these showers result from a small number of interactions of the primary particle above 10 $\mathrm{km}$ height in the atmosphere, it may be concluded that the muons observed underground correspond to the decay of fast secondaries which are produced moving

\footnotetext{
* Research supported by the National Science Foundation.
} 
forward (relative to the direction of the incoming cosmic ray primary) in the center of momentum system. Muons which are produced from secondaries moving backward in the center of the momentum system would appear at much larger distances from the shower axis and at much lower energies due to the effect of the Lorentz transformation.

It is interesting to ask whether these muons may correspond to fragments of the projectile primary cosmic ray discussed in the model of limiting fragmentation [1] or whether these muons are close enough in phase space to the primary particle to reflect some of the properties of the primary interaction which gave rise to their decay parents [2]. One piece of evidence would seem to indicate that muons observed underground are not entirely associated with the large number of pionization decay products produced which have little correlation with either the primary cosmic ray (projectile) or the air nucleus (target). The ratio of positive to negative muons is greater than one over a wide range of energies (i.e., in the range 1.2 to 1.3 up to $1.5 \mathrm{TeV}$ ) indicating that the character of the primary cosmic radiation, being mostly protons, is reflected in the charge balance of single detected muons underground [3] . A preliminary study based on a sample of less than 90 muon showers where two or more muons were detected indicates, as would be expected, that the charge ratio is nearer to 1.0 for these events corresponding to primary interactions where larger numbers of secondary particles are produced [3].

Recent analyses indicate that kinematic distributions of secondaries close to the limiting forms are obtained at even currently available accelerator energies of the order of $30 \mathrm{GeV}$ [4]. Hence, comparison of results from cosmic ray studies at higher energies with those from accelerator experiments may test the rate at which the asymptotic distributions are approached.

Muon showers detected by the Utah cosmic ray experiment are recorded digitally on magnetic tape for computer analysis at a later time. The present analyses represent the selection of one set of events from these tapes with a particular objective in view. The objective was to extend the phenomenology developed for showers having small mean numbers of muons (see sect. 3; also ref. [5]) to larger showers An approximate data combination procedure was used to consolidate available muon shower data on two- and three-particle spatial correlations to standard conditions of $45^{\circ}$ zenith and $2.5 \times 10^{5} \mathrm{~g} \mathrm{~cm}^{-2}\left(E_{\mu} \simeq 1 \mathrm{TeV}\right)$ and to compare these results with new data on four-muon spatial correlations.

\section{INCLUSIVE DISTRIBUTIONS}

The objective of underground cosmic ray shower measurements is usually to relate the distributions measured to distributions produced by single interactions of the primary particle through a shower development calculation. Therefore, even though the distributions measured in the present experiment are statistical distributions summed over successive interactions of the primary cosmic ray, it will be of interest 
to examine the general character of inclusive distributions for single interactions in some detail.

Inclusive cross sections for $m$ secondaries as a function of their transverse momentum $p_{\mathrm{t}}$, their longitudinal momentum $p_{\ell}$, and the incident projectile energy $E_{p}$ may be written as

$$
\mathrm{d}^{2 m} \sigma / \prod_{m} \mathrm{~d} p_{\ell m} \pi \mathrm{d} p_{\mathrm{t} m}^{2}=f_{m}\left(E_{p}, p_{\ell 1}, p_{\mathrm{t} 1}, \ldots, p_{\ell m}, p_{\mathrm{t} m}\right) .
$$

Then we have the following possibilities: If the cross section factors completely, then

$$
f_{m} \rightarrow g_{m}\left(p_{\ell 1}, \ldots, p_{\ell m}\right) h_{m}\left(p_{\mathrm{t} 1}, \ldots, p_{\mathrm{t} m}\right) I_{m}\left(E_{p}\right) .
$$

If the hypothesis of limiting fragmentation applies [1], then

$$
\lim E_{p} \rightarrow \infty \quad f_{m} \rightarrow f_{m}\left(p_{\ell 1}, p_{\mathrm{t} 1}, \ldots, p_{\ell m}, p_{\mathrm{t} m}\right)
$$

If the hypothesis of limiting fragmentation appleis and we have factorization as well, then

$$
f_{m} \rightarrow g_{m}\left(p_{\ell 1}, \ldots, p_{\ell m}\right) h_{m}\left(p_{\mathrm{t} 1}, \ldots, p_{\mathrm{t} m}\right)
$$

If the hypothesis of scaling applies, then

$$
\lim E_{p} \rightarrow \infty \quad f_{m} \rightarrow f_{m}\left(x_{1}, p_{\mathrm{t} 1}, \ldots, x_{m}, p_{\mathrm{t} m}\right),
$$

where $x_{m}=2 p_{\ell m} / s^{1 / 2}$. If the hypothesis of scaling applies and we have factorization as well then

$$
f_{m} \rightarrow g_{m}\left(x_{1}, \ldots, x_{m}\right) h_{m}\left(p_{t_{1}}, \ldots, p_{\mathrm{t} m}\right) .
$$

Note that in any case if $f_{m}$ factors then there are no correlations between the longitudinal and transverse momenta of secondaries. Also if the distributions of secondaries are completely uncorrelated with one another, then $f_{m} \rightarrow f_{1}^{m}$.

Statistical distributions in transverse momentum of the secondaries must take into account the energy spectrum $j\left(E_{p}\right) \mathrm{d} E_{p}$ of the primary cosmic rays. Hence the distributions for one, two, three and four particles may be written as follows:

$$
\begin{aligned}
D_{1}\left(p_{\mathrm{t}}\right) \pi \mathrm{d} p_{\mathrm{t}}^{2} \simeq & \int f_{1} j \theta\left(E_{p}-p_{\ell}\right) \mathrm{d} E_{p} \mathrm{~d} p_{\ell} \pi \mathrm{d} p_{\mathrm{t}}^{2}, \\
D_{2}\left(p_{\mathrm{rt}}\right) \pi \mathrm{d} p_{\mathrm{rt}}^{2} \simeq & \int f_{2} j \theta\left(E_{p}-p_{\ell_{1}}-p_{\ell_{2}}\right) \delta\left(\boldsymbol{p}_{\mathrm{rt}}+\boldsymbol{p}_{\mathrm{t}_{1}}-\boldsymbol{p}_{\mathrm{t}_{2}}\right) \mathrm{d} E_{\dot{p}} \\
& \times \prod_{2} \mathrm{~d} p_{\ell m} \pi \mathrm{d}_{\mathrm{t} m}^{2},
\end{aligned}
$$


and where

$$
\begin{aligned}
& D_{3}\left(p_{\mathrm{rt}_{1}}, p_{\mathrm{rt}_{2}}, p_{\mathrm{rt}_{3}}\right) \prod_{3} \pi \mathrm{d} p_{\mathrm{rt} m}^{2}, \\
& D_{4}\left(p_{\mathrm{rt}_{1}}, p_{\mathrm{rt}_{2}}, p_{\mathrm{rt}_{3}}, p_{\mathrm{rt}_{4}}, p_{\mathrm{rt}_{5}}, p_{\mathrm{rt}_{6}}\right) \prod_{6} \pi \mathrm{d} p_{\mathrm{rtm}}^{2},
\end{aligned}
$$

are constructed in a manner similar to that used to construct $D_{2}$. The notation $p_{t}$ refers to the transverse momentum relative to the shower axis while the notation $p_{\text {rt }}$ refers to the relative transverse momentum between pairs of particles.

Here we have used $\theta$ as a function which is 1.0 when its argument is greater than 0.0 and zero otherwise. The use of the $\theta$ function in this manner only provides for approximate conservation of energy because the momenta of other secondaries which have not decayed into muons observed is neglected. We also make use of the fact that $p_{\ell}>p_{\mathrm{t}}$ so $p^{2}=p_{\ell}^{2}+p_{\mathrm{t}}^{2} \simeq p_{\ell}^{2}$. The $\delta$ function relates the transverse momenta of a pair of muons to their relative transverse momentum.

\section{ANALYSIS OF UNDERGROUND MUON SHOWERS}

Interactions of the primary particles are not directly studied in the present experiment and the extent of muon showers underground is larger than any of the currently operating charged particle detector systems including the Utah detector which is the largest instrument of its kind [6]. Typical muon showers observed in the present experiment, with muons having threshold energies in the range 1 to 10 $\mathrm{TeV}$, are spread over areas of 100 to $300 \mathrm{~m}^{2}$. To make a direct observation of the way in which shower particles are distributed about the axes of muon showers at these energies, one would need a detector having good angular and spatial resolution that has dimensions of the order of $20 \mathrm{~m} \times 20 \mathrm{~m}$.

Inasmuch as no such detector now exists, we instead study the correlations between groups of particles in individual showers. What we do, in effect, is to examine the sensitive area presented by our detector at each angle and break these sensitive areas down into sets of two, three, and four small areas, such that if one muon passed through each of these areas that the group of two, three, and four muons could satisfy the triggering and aperture requirements of the detector. We compile the sums of the products of these areas as a function of detector separation, zenith, and azimuth.

Providing that the area of the shower is much larger than the area of any small detector, the probability that a muon will hit a single small detector is proportional to the product of the area of a small detector and the shower radial density distribution $\rho(R)$ evaluated at the position of that detector. The axis of the shower is defined by the initial direction of the primary cosmic ray and is maintained through successive interactions because of its high incident energy. The radial density distri- 
bution $\rho$ is the single particle statistical distribution which sums all the secondary particles produced that decay into muons observed underground above the threshold energy. The probability of detecting groups of two, three and four particles will involve products of two, three and four such factors multiplied by another factor involving the distribution of shower sizes. We assume that the dependence of data on particle separation is determined solely by products of the radial density distribution. Accordingly, we factor the sums of the products of detector sensitive areas, the average detector triggering efficiency, and write the counting rates for groups of two, three and four muons corrected for detector triggering efficiency in equivalent sets of small detectors as follows:

$$
R_{2}\left(\mathrm{~m}^{-4} \mathrm{sr}^{-1} \mathrm{sec}^{-1}\right)=N_{2} I_{2}
$$

where

where

$$
\begin{aligned}
& N_{2}=\sum M(M-1) F(M), \quad I_{2}=\int \rho(\mathrm{R} 1) \rho(\mathrm{R} 2) \mathrm{d} a, \\
& R_{3}\left(\mathrm{~m}^{-6} \mathrm{sr}^{-1} \mathrm{sec}^{-1}\right)=N_{3} I_{3},
\end{aligned}
$$

$$
N_{3}=\sum M(M-1)(M-2) F(M), \quad I_{3}=\int \rho(\mathrm{R} 1) \rho(\mathrm{R} 2) \rho(\mathrm{R} 3) \mathrm{d} a,
$$$$
R_{4}\left(\mathrm{~m}^{-8} \mathrm{sr}^{-1} \mathrm{sec}^{-1}\right)=N_{4} I_{4}
$$

where

$$
\begin{aligned}
& N_{4}=\sum_{M(M-1)(M-2)(M-3) F(M),} \\
& I_{4}=\int \rho(\mathrm{R} 1) \rho(\mathrm{R} 2) \rho(\mathrm{R} 3) \rho(\mathrm{R} 4) \mathrm{d} a .
\end{aligned}
$$

The factors of $M$ multiplying the shower size distribution $F(M)$ take into account the number of ways that $M$ muons in a shower may be chosen to fall into two, three and four detectors, respectively. The integrals are over the areas, $\mathrm{d} a$, where shower axes fall and the sums over shower sizes.

\section{RELATIONSHIP BETWEEN STATISTICAL DISTRIBUTIONS OF SECONDARIES IN TRANSVERSE MOMENTUM AND THE DISTRIBUTIONS IN SHOWER SIZES AND SHOWER RADIAL DENSITIES}

If we consider the hypothetical case of showers produced by single interactions at fixed atmospheric height, the distributions developed in sects. 2 and 3 must be equivalent since $p_{\mathrm{t}} \propto r$. Integrating these distributions must yield the total rates. Hence, we would expect the integrals below to be proportional to powers of the mean numbers of secondaries produced multiplied by an intensity factor. Therefore, 


$$
\begin{aligned}
& \int D_{1}\left(p_{\mathrm{t}}\right) \pi \mathrm{d} p_{\mathrm{t}}^{2} \propto\langle m\rangle, \quad \int D_{2}\left(p_{\mathrm{rt}}\right) \pi \mathrm{d} p_{\mathrm{rt}}^{2} \propto\left\langle m^{2}\right\rangle, \\
& \int D_{3}\left(p_{\mathrm{rt}_{1}}, p_{\mathrm{rt}_{2}}, p_{\mathrm{rt}_{3}}\right) \prod_{3} \pi \mathrm{d} p_{\mathrm{rtm}}^{2} \propto\left\langle m^{3}\right\rangle, \\
& \int D_{4}\left(p_{\mathrm{rt}_{1}}, \ldots, p_{\mathrm{rt}_{6}}\right) \prod_{6} \pi \mathrm{d} p_{\mathrm{rtm}}^{2} \propto\left\langle m^{4}\right\rangle .
\end{aligned}
$$

In a similar way, the cosmic ray phenomenology gives (to the highest order in $M$ )

$$
\begin{aligned}
& \int R_{1} \mathrm{~d} a=\sum M F(M) \int \rho \mathrm{d} a \propto\langle M\rangle, \\
& \int R_{2}(x) \pi \mathrm{d} x^{2}=\sum M(M-1) F(M) \iint \rho \rho \mathrm{d} a \pi \mathrm{d} x^{2} \propto\left\langle M^{2}\right\rangle, \\
& \int R_{3}\left(x_{1}, x_{2}, x_{3}\right) \prod_{3} \pi \mathrm{d} x_{m}^{2} \\
& \quad=\sum M(M-1)(M-2) F(M) \iint \rho \rho \rho \mathrm{d} a \prod_{3} \pi \mathrm{d} x_{m}^{2} \propto\left\langle M^{3}\right\rangle, \\
& \int R_{4}\left(x_{1}, \ldots, x_{6}\right) \prod_{6} \pi \mathrm{d} x_{m}^{2} \\
& \quad=\sum M(M-1)(M-2)(M-3) F(M) \iint \rho \rho \rho \rho \mathrm{d} a \prod_{6} \pi \mathrm{d} x_{m}^{2} \propto\left\langle M^{4}\right\rangle .
\end{aligned}
$$

Here the $x$ are the spatial separations between pairs of particles.

Notice also that the energy spectrum of the primary cosmic rays $j$ and the integral over energy of these primaries enters only once in each of the distributions $D$. In a similar way, the distribution of shower sizes $F$ enters only once in each of the rates $R$ as part of a factored sum. No dependence on longitudinal momentum appears explicitly in the rates $R$. Inasmuch as the shower sizes produced will be a function of the incident energy, the above character of the rates suggests that the single interaction distributions factor into a term dependent on $E_{p}$ and $p_{\ell}$ and one which is dependent on $p_{\mathrm{t}}$ and $p_{\ell}$. Both of these are then integrated over $p_{\ell}$. The assumption that the correlation integrals may be written as products of the radial density distribution $\rho$ is equivalent to assuming that the data may be represented by uncorrelated single particle inclusive distributions, i.e.,

$$
f_{m} \rightarrow f_{1}^{m}=k_{1}^{m}\left(p_{t}, p_{\ell}\right) l_{1}^{m}\left(E_{p}, p_{\ell}\right) .
$$

In reality, the muon showers observed underground result from a small number of interactions of the cosmic ray primary rather than from a single interaction. In view of the small number of interactions, however, it is unlikely that strongly correlated single interaction distributions could produce observed showers described by uncorrelated distributions. Therefore, we would expect that the qualitative features of the above discussion should be valid for actual showers too. 


\section{EMPIRICAL RULES TO CONSOLIDATE THE DATA}

Shower data for this study has been collected spanning the range of zenith angles from 40 to $70^{\circ}$ and slant depths of rock from 2 to $6.5 \times 10^{5} \mathrm{~g} \mathrm{~cm}^{-2}$ in a running time of 18509814 seconds. The empirical consolidation procedure is based on the observation that the measured rates of shower events at fixed zenith and slant depths are relatively insensitive to variation in azimuth, but that shower rates are attenuated at fixed zenith and varying slant depth in roughly the same manner as are vertical measurements of single muon intensities. The other necessary ingredient obtained from the behavior of the correlation integrals, $I_{2}=\int \rho \rho \mathrm{d} a, I_{3}=\int \rho \rho \rho \mathrm{d} a$, and $I_{4}=\int \rho \rho \rho \rho \mathrm{d} a$, described in the previous section. Measurements of two- and three-particle correlations as a function of zenith angle and slant depth have been found to be in qualitative agreement with those predicted on the basis of an empirical radial density distribution. This empirical representation, which decreases a little less rapidly than exponentially with distance from the shower axis has been parametrized as the product of a polynomial

$$
\begin{aligned}
P(R / \sigma)= & \left(3.7988991 \times 10^{-2}\right)+\left(-2.7728861 \times 10^{-2}\right)(R / \sigma) \\
& +\left(1.3724899 \times 10^{-2}\right)(R / \sigma)^{2}+\left(-3.7617982 \times 10^{-3}\right)(R / \sigma)^{3} \\
& +\left(5.7263392 \times 10^{-4}\right)(R / \sigma)^{4}+\left(-4.695767 \times 10^{-5}\right)(R / \sigma)^{5} \\
& +\left(1.4005871 \times 10^{-6}\right)(R / \sigma)^{6}
\end{aligned}
$$

times an exponential,

where

$$
\rho(r)=P(r / \sigma) \mathrm{e}^{-(r / \sigma)}
$$

$$
\sigma=(3.6 \pm 0.4 \mathrm{~m}) \sec ^{1.3} \theta / E_{\mu}^{0.8},
$$

and $\theta$ and $E_{\mu}$ are the zenith angle in degrees and threshold muon energy in units of $\mathrm{TeV}$ respectfully [5]. Threshold energies are estimated from the slant depth of rock traversed using a range energy relation with $b=3.5 \times 10^{-6} \mathrm{~cm}^{2} \mathrm{~g}^{-1}$ [7]. Accordingly, the data for two, three and four particle events are adjusted to standard conditions of $45^{\circ}$ zenith and $2.5 \times 10^{5} \mathrm{~g} \mathrm{~cm}^{-2}$ slant depth $\left(E_{\mu}\right.$ approximately $\left.1 \mathrm{TeV}\right)$ using ratios of products of the vertical depth vs. intensity function of single muons and of the correlation integrals evaluated using the empirical radial density distribution.

The results of the consolidation are shown in fig. 1 , where three curves correspond to the counting rates for 13511 two-, 3230 three-, and 836 four-muon correlations. The binned, ordered (largest separations put first), combinations of separations used to specify the rates of groups of three and four muons may be understood simply as follows: In the case of the three-muon rates, there are three separations which are to be sorted into $2 \mathrm{~m}$ intervals. The first combination contains all the data 

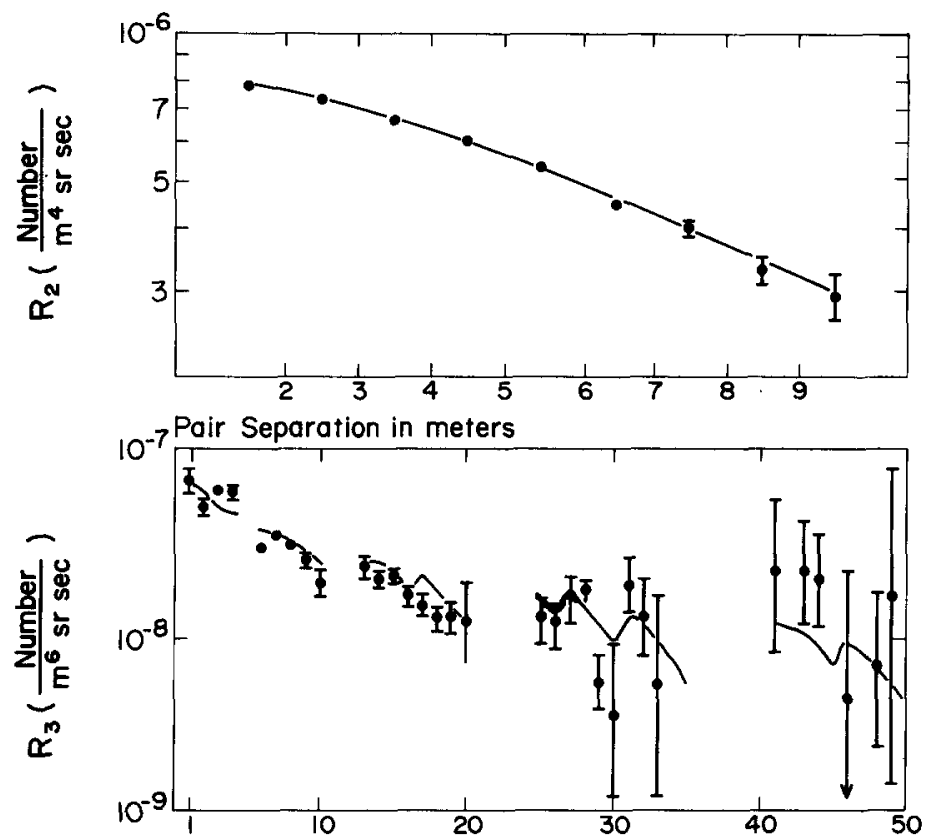

Binned, Ordered, Combinations of 2 Meter Separation intervals

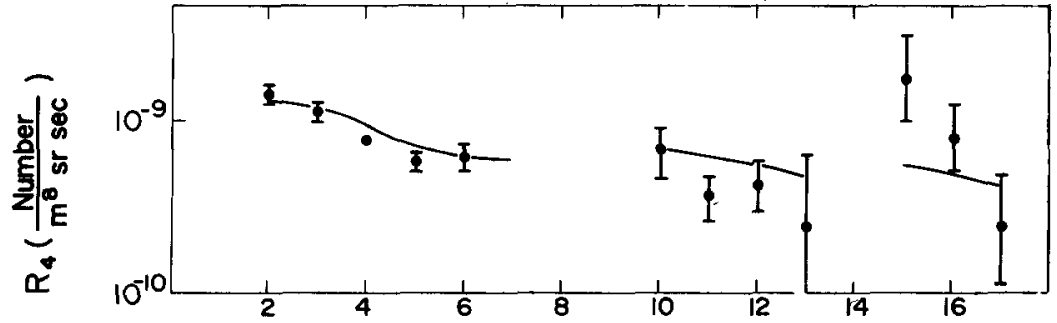

Binned, Ordered, Combinations of 4 Meter Separation Intervals

Fig. 1. Counting rates for groups of two, three and four particles as a function of separation. An empirical scaling technique has been used to consolidate data taken over a range of zenith angles from 40 to $70^{\circ}$ and a range of depths between 2 and $6.5 \times 10^{5} \mathrm{~g} \mathrm{~cm}^{-2}$ to standard conditions of $45^{\circ}$ zenith and equivalent slant depth of $2.5 \times 10^{5} \mathrm{~g} \mathrm{~cm}^{-2}\left(E_{\mu} \sim 1 \mathrm{TeV}\right)$.

where all three muons are within $2 \mathrm{~m}$ of each other. The second combination contains all the data where one separation is in the range 2 to $4 \mathrm{~m}$, and the other two are within $2 \mathrm{~m}$, etc [5]. Rates for groups of four muons are specified in a similar manner in $4 \mathrm{~m}$ separation intervals. In this case, there are six separations to be considered, of course. The solid lines are the predictions based on the empirical radial density distribution discussed above for the value of the parameter $\sigma$ giving the best fit to the data in each case. The qualitative agreement between the shapes of the measured and predicted rates is apparent. 


\section{ESTIMATES OF THE MEAN SHOWER MULTIPLICITY, THE EXPONENT OF THE SHOWER SIZE DISTRIBUTION, AND THE CHARACTERISTIC SHOWER RADIUS}

Ratios of the three normalization constants, $N_{2}, N_{3}, N_{4}$, may be used to estimate the exponent of the shower size distribution. Alternatively, these same equations may be used to derive the range of mean shower multiplicity, $\langle M\rangle$, determined by each ratio. Qualitatively speaking, the three-muon correlations correspond to showers having a higher mean number of muons than do those used for the two-muon correlations. The four-muon correlations correspond to still higher mean numbers. Therefore, the ratios of most interest to test both the dependence of the exponent of the shower size distribution on shower size and the dependence of the mean number of muons on the order of the particle correlations will be $N_{2} / I_{\mathrm{s}}, N_{3} / N_{2}$, and $N_{4} / N_{3}$. $I_{\mathrm{s}}$ is the intensity of single muons measured for the same conditions; i.e., $I_{\mathrm{s}} \simeq 8 \times 10^{-4} \mathrm{~m}^{-2} \mathrm{sr}^{-1} \mathrm{sec}^{-1}$. These ratios give rise to the following sets of equations assuming as before that $F(M) \propto M^{-\delta}$

$$
\begin{aligned}
& \frac{N_{2}}{I_{\mathrm{s}}} \frac{\sum_{M(M-1) F(M)}}{\sum_{M F(M)}}=\frac{\left\langle M^{2}\right\rangle-\langle M\rangle}{\langle M\rangle} \frac{\sum\left(M^{-\delta+2}-M^{-\delta+1}\right)}{\sum M^{-\delta+1}}, \\
& \frac{N_{3}}{N_{2}}=\frac{\sum M(M-1)(M-2) F(M)}{\sum M(M-1) F(M)}=\frac{\left\langle M^{3}\right\rangle-3\left\langle M^{2}\right\rangle+2\langle M\rangle}{\left\langle M^{2}\right\rangle-\langle M\rangle} \\
& =\frac{\sum\left(M^{-\delta+3}-3 M^{-\delta+2}+2 M^{-\delta+1}\right)}{\sum\left(M^{-\delta+2}-M^{-\delta+1}\right)} \\
& \frac{N_{4}}{N_{3}}=\frac{\sum M(M-1)(M-2)(M-3) F(M)}{\sum M(M-1)(M-2) F(M)} \\
& =\frac{\left\langle M^{4}\right\rangle-6\left\langle M^{3}\right\rangle+11\left\langle M^{2}\right\rangle-6\langle M\rangle}{\left\langle M^{3}\right\rangle-3\left\langle M^{2}\right\rangle+2\langle M\rangle} \\
& =\frac{\sum\left(M^{-\delta+4}-6 M^{-\delta+3}+11 M^{-\delta+2}-6 M^{-\delta+1}\right)}{\sum\left(M^{-\delta+3}-3 M^{-\delta+2}+2 M^{-\delta+1}\right)} .
\end{aligned}
$$

In addition, the general character of the sums requires that

$$
1<\langle M\rangle<\left\langle M^{2}\right\rangle^{1 / 2}<\left\langle M^{3}\right\rangle^{1 / 3}<\left\langle M^{4}\right\rangle^{1 / 4}
$$

Fig. 2 shows the normalization constants, $N$, of the fits to the rate curves of fig. 1 , 


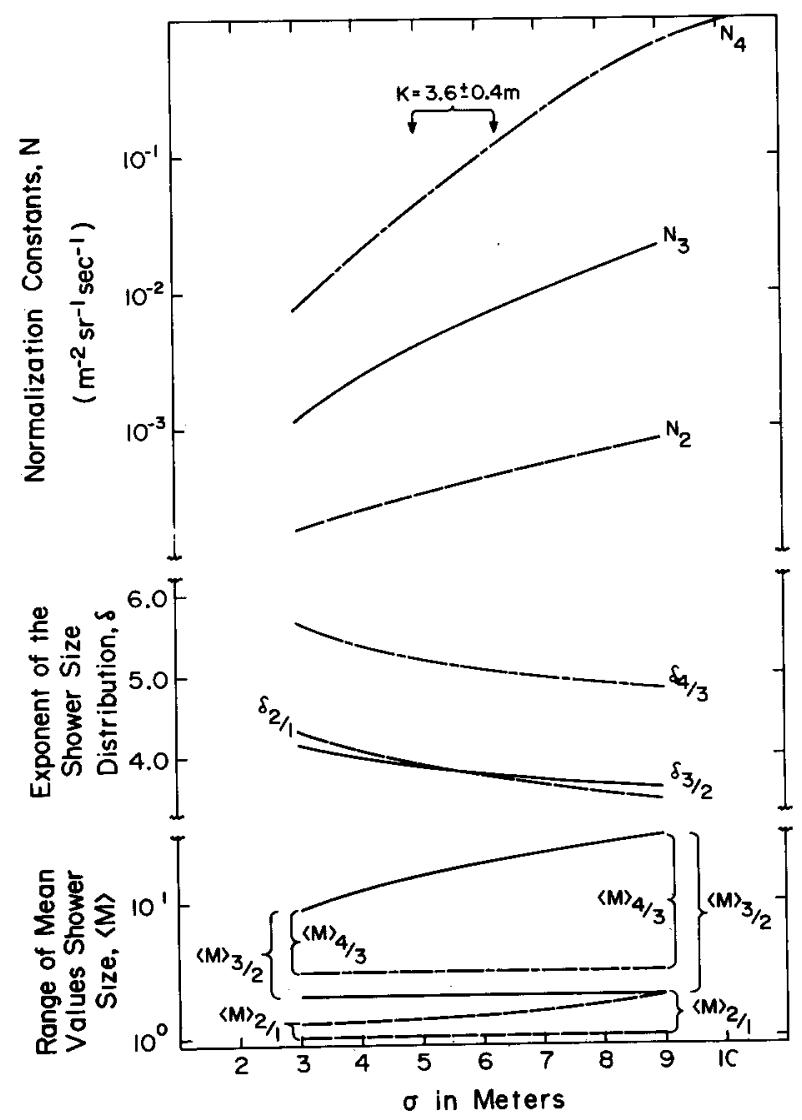

Fig. 2. $N, \delta$, and $\langle M\rangle$ as a function of the parameter $\sigma$ of the empirical radial density distribution.

the estimates of the exponent of the shower size distribution, $\delta$, and the range of mean values of shower sizes, $\langle\boldsymbol{M}\rangle$, determined by taking ratios of the normalization constants in these fits as a function of the fitting parameter, $\sigma$.

The fits giving the best agreement between the shapes of the rate curves measured (see fig. 1) and predicted were as follows: For the two-particle correlations, $\sigma=5.3$ $\mathrm{m}$; for the three-particle correlations, $\sigma=6 \mathrm{~m}$; and for the four-particle correlations, $\sigma \simeq 9 \mathrm{~m}$. As indicated by the bracket and arrows at the top of fig. 2 , the fits to the two- and three-particle correlations are obtained for values of $\sigma$ which are in the range of this parameter determined by fitting two-particle correlations at individual zenith angles and slant depths. The best fit to the four-particle correlations occurs at a larger value of $\sigma$, a point we will return to later.

The relationships between the normalization constants, the mean values of powers of $M$, and the inequalities relating mean values of powers of $M$ do not allow us to 
assign a unique mean value of $M$ corresponding to showers contributing to each ratio. They do allow us to assign the range of mean values, and we see in fig. 2 that the range of values of $M$ is shifted upwards as we take ratios of higher order correlations. It is seen that estimates of the exponent of the shower size distribution obtained from the ratio of four-particle correlations to three-particle correlations are almost one power steeper than those obtained from the ratio of three-particle correlations to two-particle correlations and two-particle correlations to single-particle intensities.

\section{CRITIQUE OF THE METHOD OF ANALYSES}

Although these qualitative features are apparent, the data are not sufficient to permit a detailed understanding of the dependence of the empirical distributions of shower size and radial density on the mean number of muons in the showers. The procedure used to consolidate the data makes use of estimates of $\rho$ which are not taken to be functions of $M$ and is, therefore, approximate. In addition, the distribution of shower sizes $F(M)=I_{0} M^{-\delta}$ and we have not taken into account in our scaling either the dependence of $I_{0}$ on zenith angle or than on threshold energy which is not factored by the vertical depth vs. intensity function of single muons or the variation in the sums $N$ resulting from the dependence of $\delta$ on zenith angle, threshold energy, and $M$. The consolidation procedure will have the largest inaccuracy in the case of the four-muon correlations, if indeed the radial density distribution is becoming broader with increasing mean number of muons.

Compensating for this, in part, is the fact that the distributions of data are peaked at small values of zenith angle and threshold energy (see table 1). Fully $65 \%$ of the four-muon correlation data falls in the range of zenith angles between 40 and $50^{\circ}$ and slant depths of 2 to $3 \times 10^{5} \mathrm{~g} \mathrm{~cm}^{-2}$. The largest correction factor comes from ratios of the depth vs. intensity function evaluated at the center depth of the various bins and at $2.5 \times 10^{5} \mathrm{~g} \mathrm{~cm}^{-2}$, while the ratios of the values of correlation integrals are much smaller, being less than the statistical uncertainties in the four-particle correlation data.

As further check, the consolidated rate curves were compared to rate curves in the individual zenith angle and depth bins which were centered close to $45^{\circ}$ and $2.5 \times 10^{5} \mathrm{~g} \mathrm{~cm}^{-2}$. These curves appeared to be in essential agreement taking into account, of course, that the centering in zenith and slant depth (see table 1) was not always exactly the same and the larger statistical uncertainties of the smaller data sample. An important point to make then, is that the data curves presented are essentially independent of the phenomenology proposed, i.e., they may be fit independently by the predictions of any theory. The sums of products of areas used to compile the rates for twos and threes were accurately calculated but those for the fours may have errors as large as $\mathbf{1 8 \%}$. We emphasize again that we make this analysis only for the purpose of making qualitative comparisons. 
Table 1

Distributions of spatial correlation data for groups of two, three and four shower muons as a function of zenith angle and slant depth.

Table 1-1: Two particle spatial correlations

\begin{tabular}{|c|c|c|c|c|c|c|c|c|c|}
\hline & 0.81 & 1.10 & 1.45 & 1.87 & 2.38 & 2.99 & 3.73 & 4.62 & energy $(\mathrm{TeV})$ \\
\hline & 2.25 & 2.75 & 3.25 & 3.75 & 4.25 & 4.75 & 5.25 & 5.75 & depth $\left(10^{5} \mathrm{~g} \mathrm{~cm}^{-2}\right)$ \\
\hline 41.25 . & 1455 & 306 & & & & & & & \\
\hline 43.75 & 1201 & 702 & & & & & & & \\
\hline 46.25 & 837 & 1043 & & & & & & & \\
\hline 48.75 & 526 & 914 & 208 & & & & & & \\
\hline 51.25 & 124 & 1087 & 351 & & & & & & \\
\hline 53.75 & 7 & 849 & 462 & & & & & & \\
\hline 56.25 & & 503 & 477 & 57 & & & & & \\
\hline 58.75 & & 207 & 375 & 270 & 23 & & & & \\
\hline 61.25 & & & 384 & 201 & 29 & 2 & & & \\
\hline 63.75 & & & 177 & 202 & 58 & 10 & 3 & & \\
\hline 66.25 & & & 43 & 139 & 45 & 28 & 24 & 4 & \\
\hline 68.75 & & & & 54 & 56 & 39 & 21 & 7 & \\
\hline \multicolumn{10}{|c|}{ Zenith angle (degrees) } \\
\hline
\end{tabular}

Table 1-2: Three particle spatial correlations

$\begin{array}{rrrrrl} & 1.0 & 1.7 & 2.6 & 4.3 & \text { energy }(\mathrm{TeV}) \\ & 2.5 & 3.5 & 4.5 & -5.5 & \text { depth }\left(10^{5} \mathrm{~g} \mathrm{~cm}^{-2}\right) \\ 42.5 & 1004 & & & & \\ 47.5 & 778 & 51 & & & \\ 52.5 & 454 & 208 & & & \\ 57.5 & 161 & 266 & & & \\ 62.5 & & 206 & 15 & & \\ 67.5 & & 29 & 23 & 33 & \end{array}$

Zenith angle (degrees)

Table 1-3: Four particle spatial correlations.

$\begin{array}{llll} & 1 & 1.7 & \text { energy }(\mathrm{TeV}) \\ & 2.5 & 3.5 & \text { depth }\left(10^{5} \mathrm{~g} \mathrm{~cm}^{-2}\right) \\ 45 & 541 & 0 & \\ 55 & 295 & 0 & \\ 65 & 0 & 0 & \end{array}$

Zenith angle (degrees) 


\section{COMPARISON OF THE GENERAL FEATURES OF COSMIC RAY AND ACCELERATOR DATA}

It is difficult to know in advance which kinematic variables will provide the best description of inelastic hadronic interactions producing large numbers of secondary particles. Individual events have considerable complexity, and a complete description would require a detailed understanding of complex higher order cross sections. Accordingly, the use of simpler statistical distributions describing the average behavior is very attractive. In the case of the present study of muon showers underground, the measurement of statistical distributions rather than the distributions corresponding to individual events is, in fact, a necessity because the complete shower is not observed.

It has been proposed that cross sections at high laboratory energies $E$ scale and become functions only of the transverse momentum, $p_{t}$, and $X=2 p_{\ell} / s^{1 / 2}$ where $p_{\ell}$ is the longitudinal momentum and $s$ is the square of the c.m. energy. The following relation which was worked out assuming scaling relates the mean transverse momentum of secondaries to the asymptotic value of the mean transverse momentum, giving

$$
\left\langle p_{\mathrm{t}}\right\rangle=\left\langle p_{\mathrm{t}} \text { asymptotic }\right\rangle\left\{1-\frac{\lambda}{\ln (E / m)}\right\}
$$

where $m$ is the nucleon mass. From accelerator data, Bali et al. [4] have estimated the coefficient $\lambda$ to be 0.58 and the asymptotic value of the mean transverse momentum to be $0.39 \mathrm{GeV} / c$. If we turn their argument around and take the asymptotic value of the mean transverse momentum from measurements of the pair correlations of muon showers [8], which was determined to be in the range between 0.5 to $0.6 \mathrm{GeV} / c$, then we would estimate that $1.2<\lambda<1.7$. This comparison with cosmic ray data at $2 \times 10^{5} \mathrm{GeV}$ indicates that the transverse momentum distribution has not reached its limiting form at $30 \mathrm{GeV}$.

On the other hand, multi-particle statistical distributions do, in fact, appear to have a common character, if not a common shape. Analysis of $\mathrm{d} \sigma / \mathrm{d} p_{\ell}$ distributions for $\pi^{-}$produced in pp collisions resulting in four-, six;, and eight-prong bubble chamber events yield essentially the same cross sections [1]. From this and the fact that the mean value of the transverse momentum is observed to be almost constant at a fraction of $\mathrm{a} \mathrm{GeV} / c$ over a wide range of $p_{\ell}$, it may be argued that nearly the same single-particle distributions in $p_{\mathrm{t}}$ and $p_{\ell}$ describe events of varying multiplicity as long as the multiplicity is small. This insensitivity of the single-particle distributions to $m$ for small values of $m$ probably explains why two- and three-particle correlations among shower muons may be fit by a single radial density distribution which is independent of $M[5]$.

Analyses of statistical distributions of secondaries by several groups have led to the proposal that the inelastic cross sections factor into products of terms which are separately functions of single variables. Fits were made to available accelerator data 
to determine the form of the factored terms. Although the numerical estimates obtained from the various fits may in some instances be rather similar, the representations employed are not equivalent. Bali et al. [4] have proposed that the inelastic inclusive cross section may be represented by the form $\mathrm{d}^{2} \sigma / \pi \mathrm{d} p_{\mathrm{t}}^{2} \mathrm{~d} p_{\ell}$ which scales at high energies and becomes $\propto F\left(p_{t}\right) G(x)$. An analysis by Akerlof et al. [9] in terms of different variables leads to the conclusion that the differential cross section factors as

$$
\mathrm{d}^{2} \sigma / \mathrm{d} p \mathrm{~d} \Omega=F^{\prime}\left(p_{\mathrm{t}}\right) G^{\prime}\left(p_{\ell}\right)
$$

where $p$ is the magnitude of the momentum of the secondary. A transformation of variables relating the differential cross sections shows that these two forms differ by a factor of $p^{-2}$.

In accelerator experiments, it is natural to study the distributions in longitudinal momentum of secondaries produced at a fixed incident energy. In contrast, underground cosmic ray experiments like the present one detect muons at fixed threshold energies which are produced by a distribution of incident primary energies. Although it is difficult to make exact comparisons, the phenomenology discussed in sect. 4 suggests that the single interaction statistical distributions are uncorrelated and factored for the cosmic ray case in agreement with the gross features, at least, of the accelerator results.

If the inelastic cross sections do have approximate factorization in the transverse momentum variable, then comparison of underground muon shower data with the predictions of theory provides an experimental test of the transverse momentum distribution of secondaries produced in interactions at mean energies of hundreds of $\mathrm{TeV}$. Parametrizations of the transverse momentum distribution suggested by Cocconi, Koester, and Perkins [10], Elbert et al. [11], and Aly et al. [12], have been tested against pair correlation data with the result that fits are obtained for all these rather different distributions corresponding to mean values of the transverse momentum of the order of $0.5 \mathrm{GeV} / c$ [13]. We anticipate that agreement with the distributions proposed by Akerlof et al. [9] and Bali et al. [4] may be achieved by adjusting the parameters so that these predicted distributions have the same mean values. In this kind of analysis, the cosmic ray data serve the useful function of determining the range of allowable parameters in each transverse momentum distribution considered. More importantly, fits to spatial correlations of muon shower pairs via a shower development calculation do favor transverse momentum distributions that have an exponential character over those that have a Gaussian character [13]. This result is in contradiction with the findings of accelerator studies which have favored distributions that have a Gaussian dependence on transverse momentum [9]. It is important to keep in mind, however, that cosmic ray data deal with nucleon-air nucleus interactions while the accelerator studies deal with nucleonnucleon collisions. Also, accelerator distributions are often measured at fixed $x$ or $p_{\ell}$, while the cosmic ray data refer to an integral over a range of these variables. 
More detailed accelerator studies are needed to resolve this question.

It is interesting to note that one of the exponential forms, the Elbert [11] form, of the transverse momentum distribution follows from statistical thermodynamic models [14] but that this form is reached only in the limiting case of large transverse momentum $[15]$; i.e., $p_{\mathrm{t}}>1 \mathrm{GeV}$. Numerically, however, the limiting form is very little different from its parent distribution at $0.5 \mathrm{GeV} / c$ and the thermodynamic predictions appear to be a good representation of the cosmic ray results.

Although only threshold energies of the component muons may be inferred from measurements of slant depth of rock penetrated in the present experiment, the measurements do have a limited sensitivity to the longitudinal momenta of the secondaries and to the energy of the primary cosmic ray. The distributions of two-, three-, and four-particle spatial correlations at fixed threshold energy each correspond to limited bands of secondary longitudinal momenta which may be determined by a shower development calculation. In addition, the median primary energy is shifted upward with increasing order of the correlation. We can understand this last effect in a rather simple way in terms of the notation developed in sect. 2 . It is not unreasonable to suppose that the single particle statistical distribution is up to some point a monotonically increasing function of incident energy and the energy spectrum of the primary cosmic rays is known to fall approximately like a power law in energy. Then, if the data are described by distributions which are approximately uncorrelated, $f_{2} \rightarrow f_{1}^{2}$, etc., and it is seen that the contributions of the integrals over energy to $D_{2}, D_{3}$, etc., occur at successively higher mean values of energy.

\section{MODEL ANALYSFS OF SHOWER DEVELOPMENT}

The measurements of underground cosmic ray detectors are related to the physics of single interactions of the cosmic ray primary through a shower development calculation. Although many such calculations have been done, few are relevant to the present experiment because they have either been one dimensional in nature (i.e., neglect the structure of the muon showers and calculate only single muon intensities) or have been calculated for a different range of threshold energies. For these reasons, as well as others, it is worthwhile to consider in some detail the results of the shower development calculations based on the conservative model put forth by the Durham, England group [16] .

The basic features of the conservative model analysis are the following:

(i) The average inelasticity for nucleons is 0.5 and for pions is 1.0 , fluctuations in the nucleon inelasticity are taken into account by a distribution of Brooke et al.

(ii) Secondary particles are assumed to be mainly pions with almost equal numbers of $\pi^{+}, \pi^{-}$, and $\pi^{0}$ mesons, consistent with iso-spin conservation.

(iii) Multiplicity distributions for pions produced in interactions of the primary cosmic ray as well as those produced by interactions of secondary pions are represented by power laws in the incident particle energy. 
(iv) The energy and transverse momentum distributions of secondaries are presented independently by empirically derived single particle statistical distributions.

(v) The mean free path of nucleons is taken to be $80 \mathrm{~g} \mathrm{~cm}^{-2}$ and that of pions to be $120 \mathrm{~g} \mathrm{~cm}^{-2}$.

Agreement between predictions of the conservative model for showers having small mean numbers of muons and measurements of muon showers incident in a range of zenith angles between 30 and $75^{\circ}$ and threshold energies in the range 0.5 to $8 \mathrm{TeV}$ has been impressive. The measured and predicted shower density spectra $[17,18]$ and shower radial density distributions $[5,13]$ are found to be in reasonable qualitative agreement both in regard to shape and dependence on zenith angle and threshold energy. Furthermore, gross characteristics of the primary particle interaction which may be inferred from a comparison of theory and measurement are in line with reasonable extrapolations from accelerator results. The mean value of the transverse momentum of secondaries is estimated from the measured spatial correlations of pairs of muons to $b e \simeq 0.6 \pm 0.5 \mathrm{GeV} / c$ [13] and the mean number of charged secondaries is estimated from measured shower intensities to depend on primary energies $E_{p}$ as $\propto E_{p}^{1 / 4}$ (or $\ln E_{p}$ ) [19] .

From the point of view of recent theoretical analyses of the physics of single interactions, the basic approach of the conservative model seems very reasonable indeed. In the multiperipheral picture of interactions [2] where there are large numbers of particles in the final state, secondary particles at the highest energies are produced with uncorrelated kinematic distributions. This general character is reflected in the phenomenology put forward to describe spatial correlations of muons in showers (see sect. 3) but the phenomenology suggests as well that the distributions factor in primary energy and in transverse momentum providing further support for the conservative model.

In addition to the predictions above, the conservative model analyses predict that the mean shower radius shrinks with increasing primary energy [19] in apparent contradiction with the new results presented in this paper. The question remains whether these results really are in disagreement with the basic tenants of the conservative model forcing that analysis to be modified for those very large final states where hundreds of secondary particles are produced or whether agreement can be achieved by taking into account what may be basically geometrical effects.

The conservative model analysis predicts a mean shower radius that decreases with increasing primary energy because higher energy primaries are pictured as penetrating deeper into the atmosphere producing larger numbers of secondaries having greater longitudinal momenta. Although this physical picture is compelling, we must take care to examine those features of the model which relate directly to the spatial correlation measurments described in the present paper.

It is implicitly assumed in the conservative model analysis that measurements of any kind may be described by single particle statistical distributions. This is so even though in the analysis process the shower development is followed through successive interactions and counting rates in pairs of detectors are calculated directly from 
the distribution of particles at a given depth because the distributions of energy and transverse momentum put into the model are single particle statistical distributions. It is further assumed that the interaction mean free paths for the primaries and the secondaries are constants independent of energy, that the secondaries produced are either all pions or pions plus a small percentage of kaons, and that the transverse momentum distribution may be represented as a factor independent of primary energy. Although all of these assumptions seem to be quite reasonable, they by no means follow in a straightforward way from the theory of inelastic scattering processes.

The data being considered here, spatial correlations between groups of four muons in individual shower events, are quite special in that they come from larger showers than were previously accessible to detailed study and that the median primary energy for this small fraction of the total data is much higher than the bulk of the data recorded at the same threshold energy [5]. In view of this, let us reexamine the assumptions of the conservative model.

First, the transverse momentum distribution is only approximately independent of incident energy. A compilation of data from a variety of experiments of different kinds by Adcock et al. [13] shows that the mean value of the transverse momentum is a slowly increasing function of the primary energy. On this basis, we might estimate that the mean transverse momentum corresponding to the four muon spatial correlations might be as much as $20 \%$ greater than that of the two particle correlations in agreement with the general trend of the data; i.e., the mean shower radius estimated is seen to increase with the order of the correlation. Although these results are suggestive, they can not be the entire answer unless the mean transverse momentum rises more rapidly than data at lower median primaries would indicate. The fact that the shower radius seems to increase with shower size could also be explained by correlations in transverse momentum which force additional particles out to larger shower radii. Since the conservative model analysis deals only with uncorrelated distributions, it would not predict such an effect even though we would expect it to do an adequate job of describing the main bulk of the data where the number of secondaries is small. Correlations of this kind cannot be ruled out by currently available experimental results. Should further experiments prove either of these effects to be important for median primary energies greater than a few hundred $\mathrm{TeV}$, then it may be necessary to reformulate the input to shower development calculations which have assumed that the interaction products are described by factored, uncorrelated single particle statistical distributions.

Second, the increase in shower radius with increase in shower size might be explained by what are basically geometrical factors. The effect could be due to enhanced contributions at higher energies to the muon showers observed resulting from interactions of the secondaries. Since the secondaries have a transverse momentum distribution themselves, their interaction products would produce muons having a broader distribution about the shower axis. The effect could also be explained by a total inelastic cross section that increases with increasing incident energy. Experi- 
mental evidence for such an increase has been summarized by Yodh et al. [20] . Since the associated median primary energy is a rapidly rising function of the number of muons detected, the larger showers would be produced preferentially higher in the atmosphere allowing the component muons to appear at larger shower radii.

Finally, we comment on the possible effect of the $\mathrm{X}$ process proposed by Bergeson et al. [21] to explain the zenith angle dependence of single muon intensities. The $X$ process involves a heavy intermediate particle having a lifetime short compared to pions and kaons which decays into muons. It is unlikely that the $X$ process would effect the spatial correlations of highly multiple events because the number of such heavy intermediaries produced in a given shower would not be expected to be large.

\section{SUMMARY}

Analysis of the four muon spatial correlations discussed in this paper suggests that the shower radial density distribution becomes broader and the shower size distribution steepens with increasing mean numbers of muons. These results might be explained geometrically in terms of a total inelastic cross section that rises with energy or in terms of enhanced contributions to muon showers observed from interactions of the secondaries. Alternately, they might be explained by a transverse momentum dsitribution which broadens markedly with increasing primary energy or by correlations in transverse momentum between particles in showers having large numbers of muons. If the effect does not have a geometrical origin, then it may be necessary to reformulate the input to shower development calculations which have assumed that interaction products are described by factored, uncorre: lated single particle statistical distributions. It is argued that the $\mathrm{X}$ process proposed by Bergeson et al. [21] to explain the zenith angle dependence of single muon intensities should have a negligible effect on spatial correlations of highly multiple events.

The usefulness of underground cosmic ray shower data for making comparisons to accelerator studies and for testing the predictions of theory is limited by the fact that interactions of the primary cosmic ray are not observed directly. In particular, the data from the present experiment do not appear to be sufficient in themselves to make a clear choice between the possible mechanisms which could produce the effect observed. On the other hand, muons observed underground result from a special cut of the secondaries produced by hadronic interactions which may be difficult to study in any other way [22].

We wish to thank Professor J. Ball and Dr. S. Pinsky for helpful discussions. We are indebted to Professor A.W. Wolfendale and J.R. Wayland for their comments on preliminary versions of this work. Special thanks go to T.M. Cannon, K.H. Davis, and R.B. Ingebretsen for their contributions to computer analysis. 


\section{REFERENCES}

[1] J. Benecke, T.T. Chou, C.N. Yang, E. Yen, Phys. Rev. 188 (1969) 2159; see also T.T. Chou and C.N. Yang, Phys. Rev. 25 (1970) 1072.

[2] G.F. Chew and A. Pignotti, Phys. Rev. 176 (1970) 2112; see also A.H. Mueller, Phys. Rev. D2 (1970) 2963.

[3] H.E. Bergeson, J.W. Keuffel, M.O. Larson, G.H. Lowe, J.H. Parker, Proc. of the 11 th Int. Conf. on cosmic rays, Budapest (1969), Acta Physica Academiae Scientiarum Hungaricae, vol. II (1971); see also J.H. Parker, Ph. D. Thesis, University of Utah (1969).

[4] N.F. Bali, Lowell S.,Brown; R.D. Peccei, A. Pignotti, Phys. Rev. Lett. 25 (1970) 557.

[5] K.H. Davis, S.M. Fall, R.B. Ingebretsen, R.O. Stenerson, Phys. Rev, D4 (1971) 607.

[6] J.W. Keuffel and J.L. Parker, Nucl. Instr. 51 (1967) 29; L.K. Hilton, M.L. Morris, and R.O. Stenerson, Nucl. Instr. 51 (1967) 43; H.E. Bergeson and C.J. Wolfson, Nucl. Instr. 51 (1967) 47.

[7] P.H. Barrett, L.M. Bollinger, G. Cocconi, Y. Eisenberg, K. Greisen, Rev. Mod. Phys. 24 (1952) 133.

[8] H.E. Bergeson,.R.B. Coats, J.W. Keuffel, M.O. Larson, G.H. Lowe, J.L. Osborne, S. Ozaki, J.H. Parker and R.O. Stenerson, J. Phys. A3 (1970) 689.

[9] C.W. Akerlof, J.G. Ashbury, D.G. Crabb, J.L. Day, K.W. Edwards, N.P. Johnson, P. Kalbaci, A.D. Krish, M.P. Lin, G.J. Marmer, M.L. Marshak, J.K. Randolph, L.G. Ratner, A.L. Reed, and P. Schmueser, Phys. Rev. D3 (1971) 645.

[10] G. Cocconi, L.J. Koester and D.H. Perkins, UCRL-10022 (1961).

[11] Y.Y. Chan, J.W. Elbert, A.R. Irwin, S. Mikamo, D. Reeder, W.D. Walker and A. Weinberg, Topical Conf. on high energy collisions of hadrons, CERN, Geneva, January 15-18 (1968).

[12] H.H. Aly, M.L. Chen and M.F. Kaplon, Nuovo Cim. 31 (1964) 905.

[13] C. Adcock, R.B. Coats, J. Wdowczyk, A.W. Wolfendale, J. Phys. A3 (1970) 697.

[14] E.L. Feinburg and I.P. Ivanenko, Rapporteur talk, Proc. of the 11th Int. Conf. on cosmic rays, Budapest (1969);

R. Hagedorn, Nuovo Cim. Suppl. 3 (1965) 147;

K. Imaeda, Nuovo Cim. 48A (1967) 147;

T. Bowen and J.R. Wayland, Nuovo Cim. 48A (1967) 663.

[15] J.R. Wayland, Nuovo Cim. (1971) in press.

[16] J.F. deBeer, B. Holyoak, J. Wdowczyk and A.W. Wolfendale, Proc. Phys. Soc. 89 (1966) 567.

[17] L.G. Porter and R.O. Stenetson, J. Phys. A2 (1969) 374.

[18] C. Adcock, J. Wdowczyk and A.W. Wolfendale, J. Phys. A2 (1969) 574.

[19] C. Adcock, R.B. Coats, J. Wdowczyk and A.W. Wolfendale, J. Phys. A4 (1971) 276.

[20] Y. Pal, J.R. Wayland and G.B. Yodh, Quanta and Fields (1971), in press.

[21] H.E. Bergeson, J.W. Keuffel, M.O. Larson, E.R. Martin and G.W. Mason, Phys. Rev. Lett. 19 (1967) 1487;

H.E. Bergeson, J.W. Keuffel, M.O. Larson, G.W. Mason and J.L. Osborne, Phys. Rev. Lett. 21 (1968) 1089,

[22] P.M. Fishbein and J.S. Trefil, Phys. Rev. D3 (1971) 238. 